\title{
Integrated modeling of the GMT Laser Tomography Adaptive Optics System
}

\author{
Piotr Piatrou ${ }^{a}$ \\ ${ }^{a}$ Australian National University, Research School of Astronomy and Astrophysics, \\ Canberra, Australia.
}

\begin{abstract}
Laser Tomography Adaptive Optics (LTAO) is one of adaptive optics systems planned for the Giant Magellan Telescope (GMT). End-to-end simulation tools that are able to cope with the complexity and computational burden of the AO systems to be installed on the extremely large telescopes such as GMT prove to be an integral part of the GMT LTAO system development endeavors. SL95, the Fortran 95 Simulation Library, is one of the software tools successfully used for the LTAO system end-to-end simulations. The goal of SL95 project is to provide a complete set of generic, richly parameterized mathematical models for key elements of the segmented telescope wavefront control systems including both active and adaptive optics as well as the models for atmospheric turbulence, extended light sources like Laser Guide Stars (LGS), light propagation engines and closed-loop controllers. The library is implemented as a hierarchical collection of classes capable of mutual interaction, which allows one to assemble complex wavefront control system configurations with multiple interacting control channels. In this paper we demonstrate the SL95 capabilities by building an integrated end-to-end model of the GMT LTAO system with 7 control channels: LGS tomography with Adaptive Secondary and on-instrument deformable mirrors, tip-tilt and vibration control, LGS stabilization, LGS focus control, truth sensor-based dynamic noncommon path aberration rejection, pupil position control, SLODAR-like embedded turbulence profiler. The rich parameterization of the SL95 classes allows to build detailed error budgets propagating through the system multiple errors and perturbations such as turbulence-, telescope-, telescope misalignment-, segment phasing error-, non-common path-induced aberrations, sensor noises, deformable mirror-to-sensor mis-registration, vibration, temporal errors, etc. We will present a short description of the SL95 architecture, as well as the sample GMT LTAO system simulation results.
\end{abstract}

\section{SL95 RATIONALE}

End-to-end computer simulations have become an integral part of the development of Adaptive Optics (AO) systems. The simulations allow to estimate the AO system performance, guide the designer in the hardware choices, compare different AO modes of operation (Single-Conjugate, Multiple-Conjugate, Multiple-Object, etc.), do system debugging or data reduction tool testing. ${ }^{1}$ The importance of $\mathrm{AO}$ simulations and the corresponding simulation tools grows even higher at the advent of the Extremely Large Telescopes (ELTs) for which AO becomes an indispensable part. The goal of this work is to present a new end-to-end simulation tool, the Fortran 95 Simulation Library (SL95), which has been created and is being successfully used for development of the Laser Tomography Adaptive Optics (LTAO) system ${ }^{2}$ for the Giant Magellan Telescope (GMT). ${ }^{3}$

AO for ELTs is extremely computationally demanding, with a requirement of real-time signal processing of $\sim 10^{5}$ sensor measurements at $\sim 1 \mathrm{kHz}$ rate to drive $\sim 10^{4}$ Deformable Mirror $(\mathrm{DM})$ actuators. These requirements, however, become much milder for the $\mathrm{AO}$ simulation tools where the real-time performance is not needed and the ordinary computers have quite adequate power to run the end-to-end AO simulations. It turns out to be more important for a simulation tool to provide a complete set of reliable mathematical models of all $\mathrm{AO}$ system elements that can be connected into complex signal propagation networks adequately representing a real AO system, i.e. to provide integrated modeling. The goal of SL95 project is to create not necessarily the most computationally efficient but reasonably complete, extensible tool-set for integrated Adaptive and Active Optics (aO) modeling. SL95 is a low-cost project: no dependency on commercial software, no excessive hardware requirements, compact and clean code, rapid development cycle minimizing the (development time)/(run time) ratio. 
Fortran 95 was chosen for a number of reasons:

1. High performance of the compiled code is combined in Fortran 95 with high-level vectorized syntax allowing rapid code development.

2. Ability to write object-oriented code.

3. Large reusable code base, especially numerical libraries.

4. Relative ease of calling the code written in other languages from Fortran 95 and vice versa, which enables portability.

With portability in mind, the SL95 was written not as a monolith application but as a library of numerical methods usable in various combinations and callable not only from the Fortran 95 but also from a "glue" application, written in other high-level languages, Matlab or Python being the most obvious examples.

As a part of the GMT project, SL95 focuses primarily on modeling of the GMT LTAO system. However, in its current state SL95 allows to simulate many other AO systems for large segmented telescopes. A minimal set of the SL95 development goals can be listed as follows:

- Truly segmented telescope optical model with possibility to apply arbitrary rigid body motions and aberrations to each individual segment.

- Layered atmospheric turbulence model with wind-driven (Taylor hypothesis) dynamics and random phase screen generators for Kolmogorov and Von Karman turbulence statistics.

- Paraxial, geometrical (ray tracing) and wave propagation of light from point and extended sources through turbulence and optical elements.

- Model for extended, polychromatic light sources including Laser Guide Stars (LGS) with cone effect and spot elongation.

- Models for Shack-Hartmann wavefront sensor (SH WFS), pyramid (PWFS) and quad cell sensors with internal wave propagation.

- Linear model for segmented deformable mirrors with actuator dynamics and coupling.

- Camera model with pixelation effects, photon and read noise.

- Least Squares Single-Conjugate Adaptive Optics (SCAO) MVM-type (matrix-vector multiplication) controller, Minimum Variance LTAO MVM-type controller. Other types of controllers are planned for the future.

- SLODAR-like embedded turbulence profiler.

- System perturbation models: atmosphere turbulence, sensor noises, optics and sensor misalignments, noncommon path aberrations, telescope aberrations, vibration-induced tip/tilt jitter, control loop delays, angular and focus anisoplanatism.

- End-to-end simulation engine(s) to integrate the complete adaptive optics system model.

- Data archiving and data visualization facilities.

In the following sections we present an overview of the SL95 package structure and simulation capabilities. 
In the following sections we present an overview of the SL95 package structure and simulation capabilities.

\section{SL95 STRUCTURE}

The main purpose of any simulation software is to aid in solving the problems arising in the course of development. Thus, constant adding new features becomes natural, which makes the code open-ended. Open-endedness is effectively addressed in the object-oriented approach by encapsulating all the model parameters into the classes. This results in the effectively parameter-less high level method calls not affected by frequent changes in the model parameterizations. Although Fortran 95 does not fully support the object-oriented paradigm, it allows one to create quite effective "quasi-object-oriented" patterns. ${ }^{4}$ The classes are organized into a hierarchical structure the main feature of which is that the higher-level classes "know" how to interact with the lower-level ones. To ensure maximal re-usability the classes are made "thin" by moving computational part down to the level of "common libraries" containing more general purpose algorithms and together making the (expandable) SL95 mathematical engine. The common libraries in turn depend on the "standard libraries" of universal mathematical tools such as LAPACK or FFTW that are obtained externally. A more detailed SL95 structure description is given on the diagram below (lower position means lower level in the hierarchy).

\section{Main program.}

Since SL95 is just a library, this part should be user-defined.

Simulator classes.
DemoSim class is provided as an example of a user-defined simulation engine.

\section{Controller classes.}

MvLtao and LsScao are the classes implementing Minimum Variance LTAO and Least Squares SCAO reconstructors, respectively. SLODAR algorithm is a part of the MvLtao class. PupilReg class is the pupil registration/stabilization algorithm implementation.

\begin{tabular}{|c|c|c|c|}
\hline $\begin{array}{l}\text { Launch class, } \\
\text { Laser Launch Telescope model. }\end{array}$ & & & \\
\hline $\begin{array}{l}\text { ShSensor class, } \\
\text { Shack-Hartmann WFS model. }\end{array}$ & $\begin{array}{l}\text { LinearDm class, } \\
\text { Deformable mir }\end{array}$ & or model. & $\begin{array}{l}\text { Turbulence } \mathrm{cl} \\
\text { Atmospheric } \mathrm{t}\end{array}$ \\
\hline $\begin{array}{l}\text { FlatOptic class, } \\
\text { Segmented flat optic (phase }\end{array}$ & screen) model. & $\begin{array}{l}\text { Camera cl } \\
\text { Imaging c }\end{array}$ & a model. \\
\hline $\begin{array}{l}\text { Field class, } \\
\text { Light source model. }\end{array}$ & & & \\
\hline $\begin{array}{l}\text { Common libraries } \\
28 \text { mathematical libr }\end{array}$ & aries covering all & needs of $\mathrm{A}$ & stem modeling. \\
\hline $\begin{array}{l}\text { Standard librar } \\
\text { The externally } \\
\text { FFTW, GEOMP } \\
\text { alization, etc. }\end{array}$ & $\begin{array}{l}\text { ies. } \\
\text { upplied univers } \\
\text { ACK2 for } 2 \mathrm{D} \text { tr }\end{array}$ & $\begin{array}{l}\text { mathem } \\
\text { angulation }\end{array}$ & $\begin{array}{l}\text { libraries: BI } \\
\text { ITPLOTLIB fo }\end{array}$ \\
\hline
\end{tabular}

The guide, a stand-alone command-line utility providing Matlab-like help system functionality through reading the self-documented source code. Additionally, one can inquire about the code structure, navigate to a class or library or even compile all the information into a single manual for a current code snapshot.

The minimalistic set of SL95 classes turns out to be sufficient because each class has reach internal parameterization making it adjustable for most of simulation tasks. It is relatively easy to modify the existing classes by adding new parameters or data processing methods, which has been found to be more user-friendly in comparison to usual for the object-oriented approach class inheritance.

\section{SL95 GALLERY}

Below we present a gallery of images generated by the SL95 illustrating various aspects of the AO system modeling. The necessary explanations are given in the figure captions. 


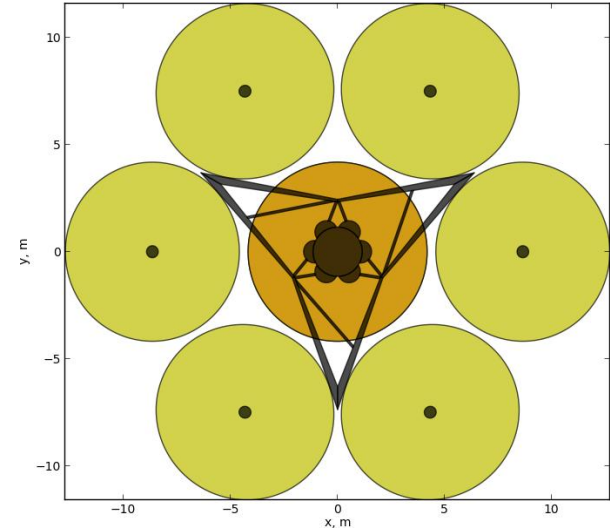

a)

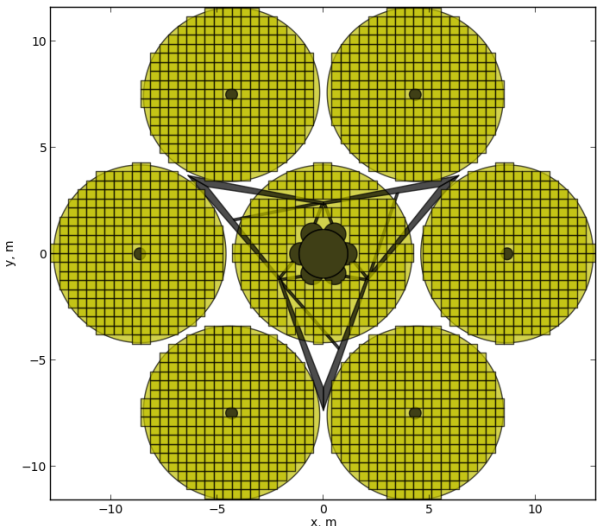

b)

Figure 1. a) Vector model of GMT pupil (combination of polygons) with realistic obscuration pattern. b) Map of 1873 active subapertures picked from a LTAO $60 \times 60$ SH WFS and superimposed onto the GMT pupil.

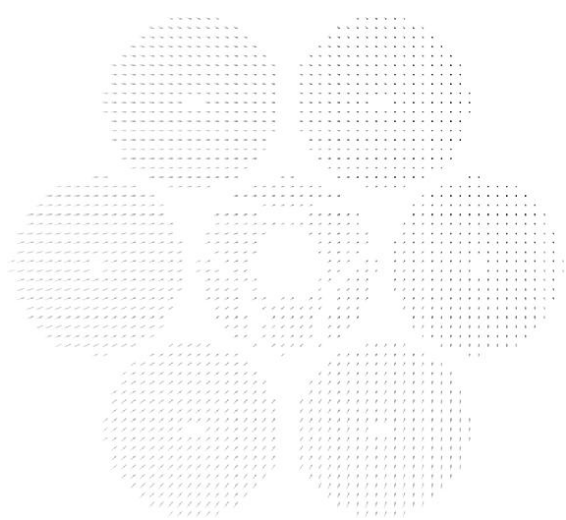

a)

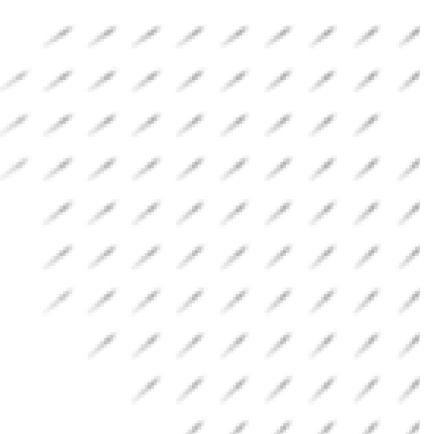

b)

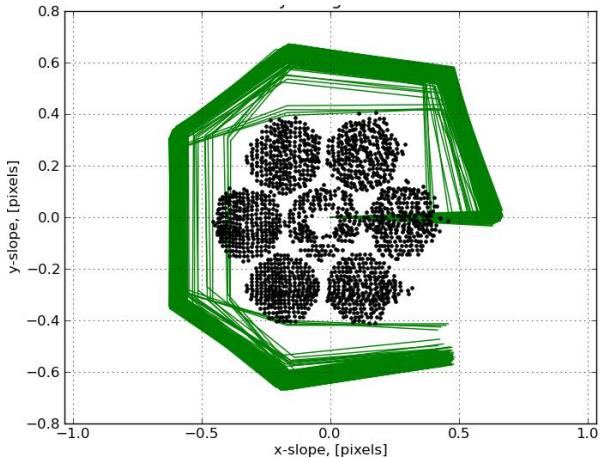

c)

Figure 2. a) Shack-Hartmann wavefront sensor camera image with LGS elongated spots. b) Zoom of the area with maximal spot elongation. c) Calibration "eye diagram" (green) of the SH WFS: trajectories of all sensor spots in response to rotating tip-tilt input. Significant irregularity is due to spot elongation. "Reference" slopes as response to zero phase are superimposed in black. 

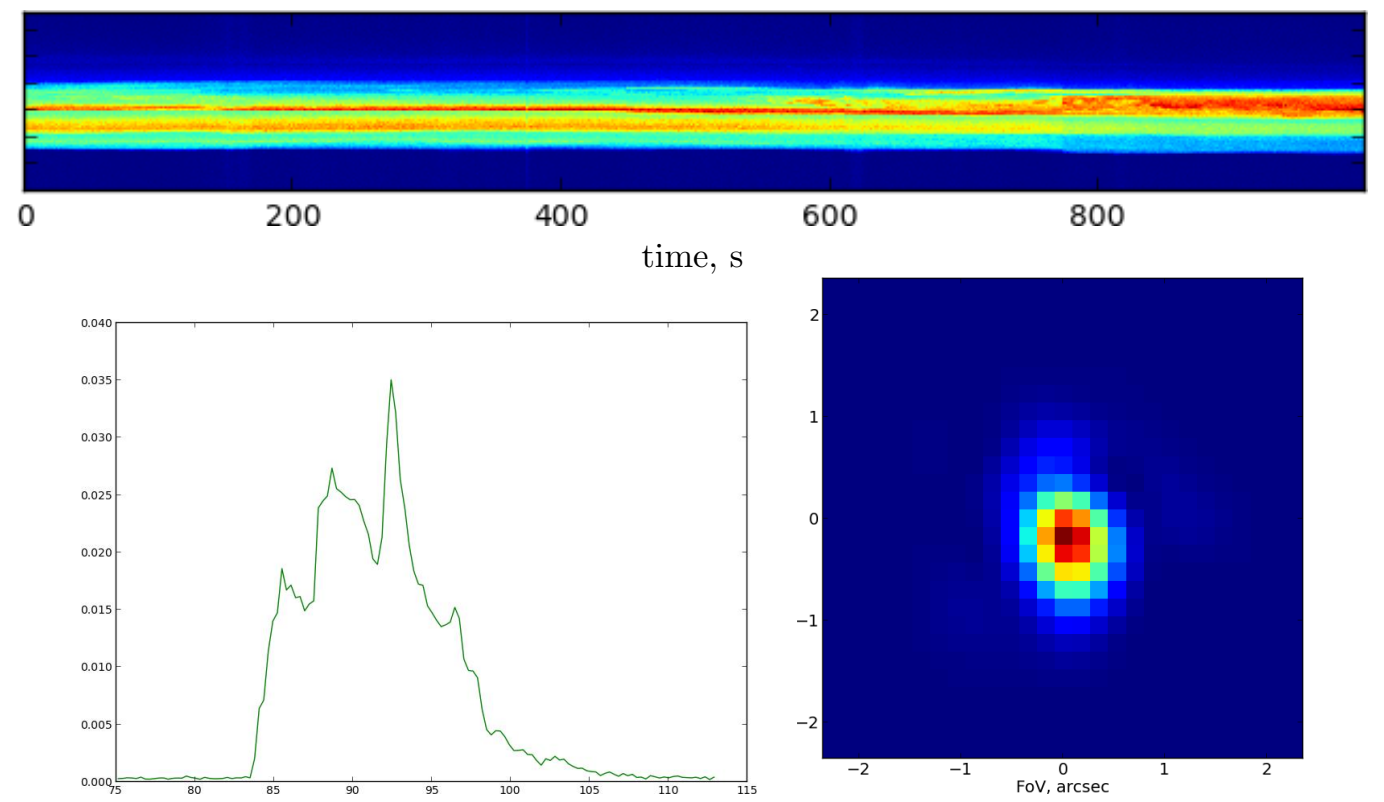

altitude, $\mathrm{km}$

Figure 3. Top: Map of remotely measured $N a$ abundance. ${ }^{5,6}$ Bottom left: $N a$ abundance cross section at $400 \mathrm{~s}$, which is used to weight the LGS longitudinal intensity profile. Bottom right: Transverse intensity distribution of the Laser Launch Telescope (LLT) beam after upward propagation to the $N a$ layer through atmospheric turbulence.

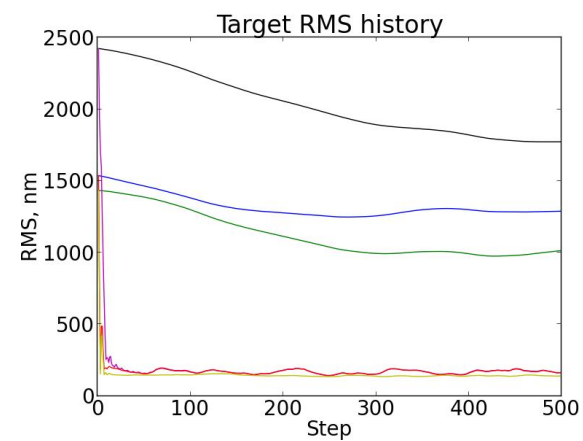

(a)

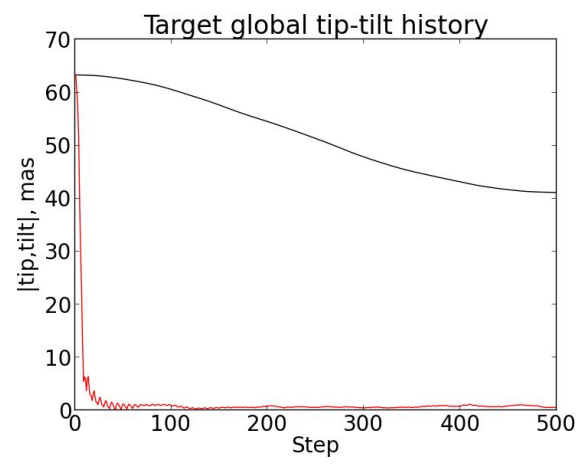

(b)

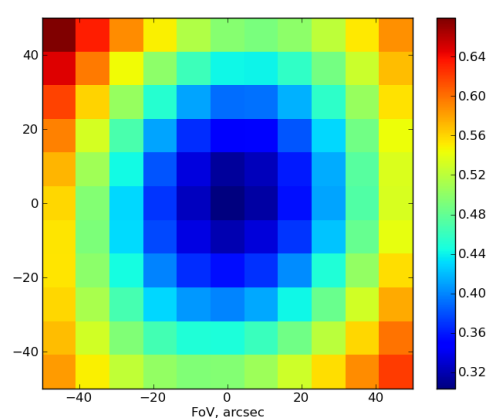

(c)

Figure 4. Time histories of the residual RMS phase (a) and global tip/tilt error (b) from the end-to-end LTAO system performance Monte-Carlo simulation. Residual RMS LTAO system error distribution (c) over the telescope field of view, piston-removed, 1-second average, transients excluded. 


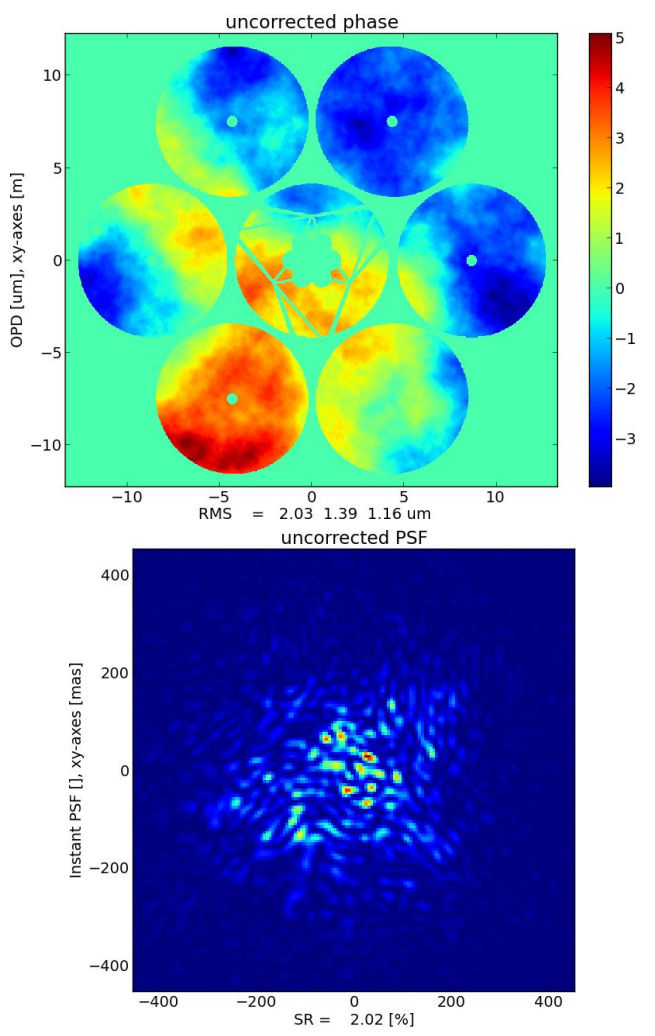

a)
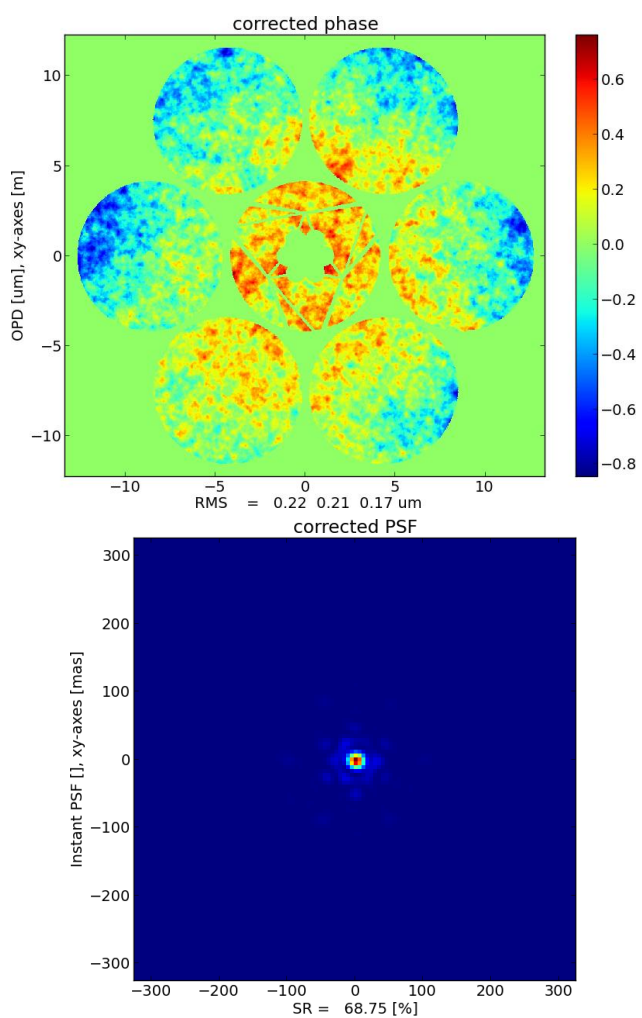

b)

Figure 5. Example of phase Minimum Variance LTAO phase correction. a) Uncorrected turbulence phase in the GMT pupil and the corresponding Point Spread Function (PSF). b) Corrected phase in the pupil and the corresponding PSF. Open-loop operation, LGS flux 800 photons $/ \mathrm{m}^{2}$ at the pupil, sensor readout noise $3 e^{-}$, standard GMT 7 -layer turbulence profile, $r_{0}=0.151 \mathrm{~m}$ at $\lambda=0.5 \mu \mathrm{m}$, zenith direction.

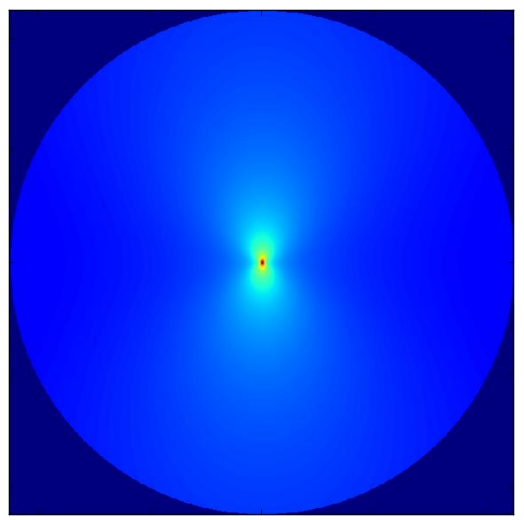

(a)

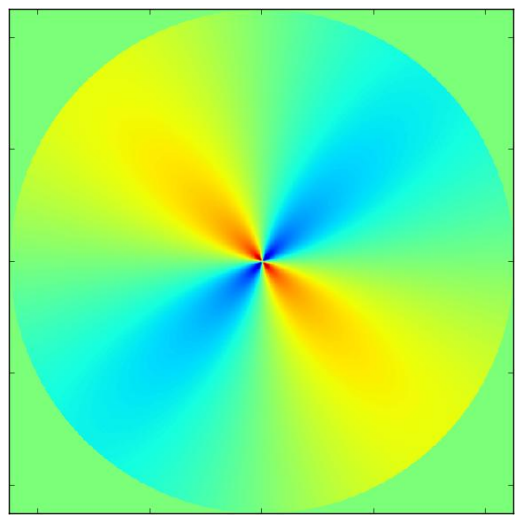

(b)

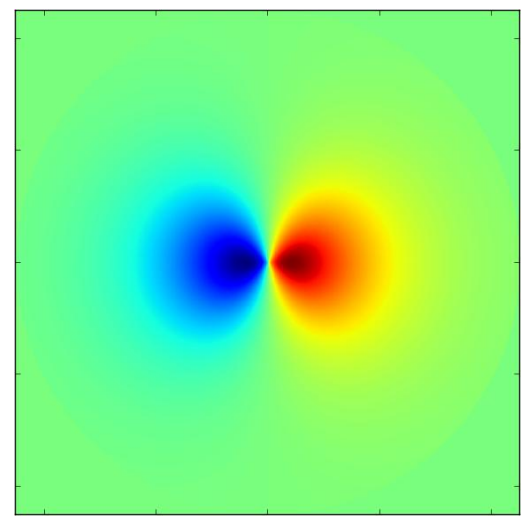

(c)

Figure 6. XX- (a), XY-slope (b) SH WFS atmospheric turbulence covariance function maps and phase-to-X-slope (c) SH WFS covariance function map for one atmospheric turbulence layer. Von Karman turbulence model with outer scale $L=60 \mathrm{~m}$. The maps are used to build the Minimum Variance LTAO reconstructor. 


\section{SKETCH OF GMT LTAO SIMULATIONS}

In this section the results of the GMT LTAO integrated model simulations are presented. We do not pretend to completeness here, we rather illustrate SL95 capabilities.

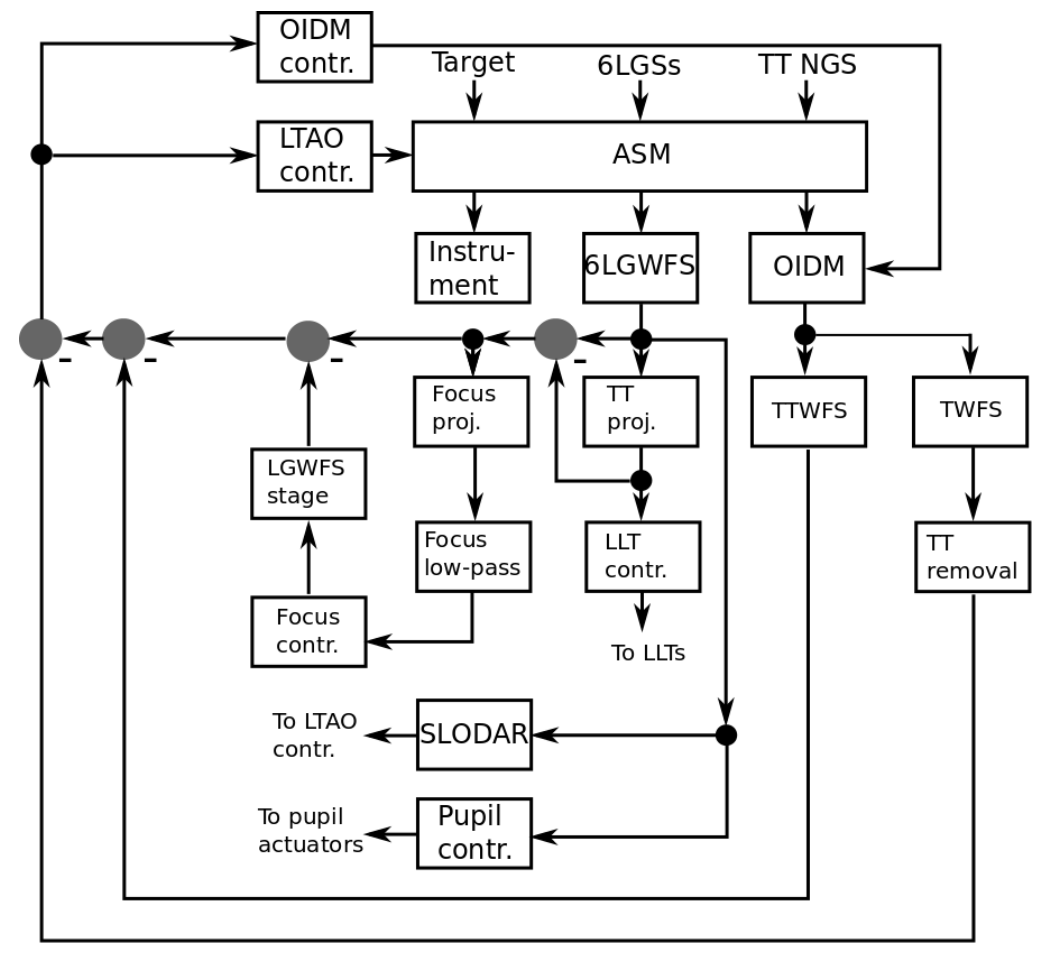

Figure 7. Simplified GMT LTAO control system diagram. Abbreviations: ASM - Adaptive Secondary Mirror, OIDM On-Instrument Deformable Mirror, TT NGS - Tip/Tilt Natural Guide Star, LGS - Laser Guide Star, LGWFS - Laser Guide WaveFront Sensor, LTAO - Laser Tomography Adaptive Optics, proj. - projection, contr. - controller, low pass low-pass temporal filter, LGWFS stage - mount for 6 LGWFS responsible also for pupil de-rotation and focus control, TTWFS - Tip/Tilt WaveFront Sensor, TWFS - Truth WaveFront Sensor, LLT - Laser Launch Telescope. SLODAR SLOpe Detection And Ranging algorithm.

A simplified model for the GMT LTAO system is presented on Fig. 7 and consists of 7 interacting control loops. Note that here we omit the control loops related to the GMT active optics such as primary mirror shaping and phasing.

1. The main channel is the subsystem consisting of 6 Shack-Hartmann wavefront sensors illuminated by 6 LGSs making regular hexagon pattern with 30" field of view offset. The sensors generate a flow of measurements being processed by the LTAO reconstructor algorithm to generate commands for the 7segment Adaptive Secondary Mirror actuators. In the simplest configuration the LTAO reconstructor provides commands to compensate all turbulence-induced aberrations except for global tip and tilt that are corrected independently by the

2. Tip-tilt correction channel: signal from a quad-cell tip-tilt wavefront sensor (TTWFS) illuminated by an off-axis Natural Guide Star is passed through the tip-tilt controller (integrator in the simplest case) and the tip-tilt correction commands are directed to the ASM. To improve the tip-tilt correction and to increase the LTAO system sky coverage, an additional, On-Instrument Deformable Mirror (OIDM) is installed between ASM and TTWFS to compensate the anisoplanatism between the scientific target and the TT NGS. Essentially, two LTAO reconstructors are working in parallel on the same set of the LGWFS measurements: one is adjusted to the scientific target direction and the other for the TT NGS direction. The difference between the two reconstructors phase estimates is directed to the OIDM. Fig. 
8 demonstrates performance of the LTAO reconstructor and tip/tilt control as a function of TT NGS offset with and without OIDM correction. Fig. 9 demonstrates the results of tip/tilt correction in case of additional large wind shake modeled for the GMT dome.
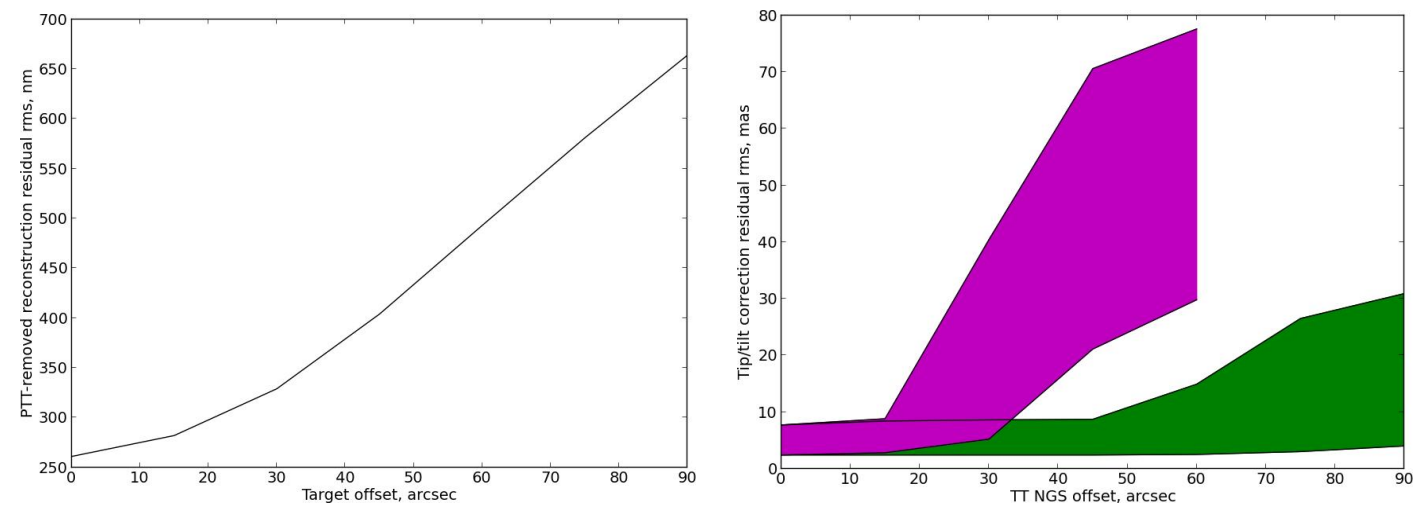

Figure 8. Left: LTAO reconstruction residual phase rms as a function of the scientific target offset. Right: residual tip/tilt rms as a function of TT NGS offset in case of OIDM correction (green) and no OIDM correction (magenta). Lower limit corresponds to TT NGS magnitude 15, upper limit corresponds to TT NGS magnitude 19.
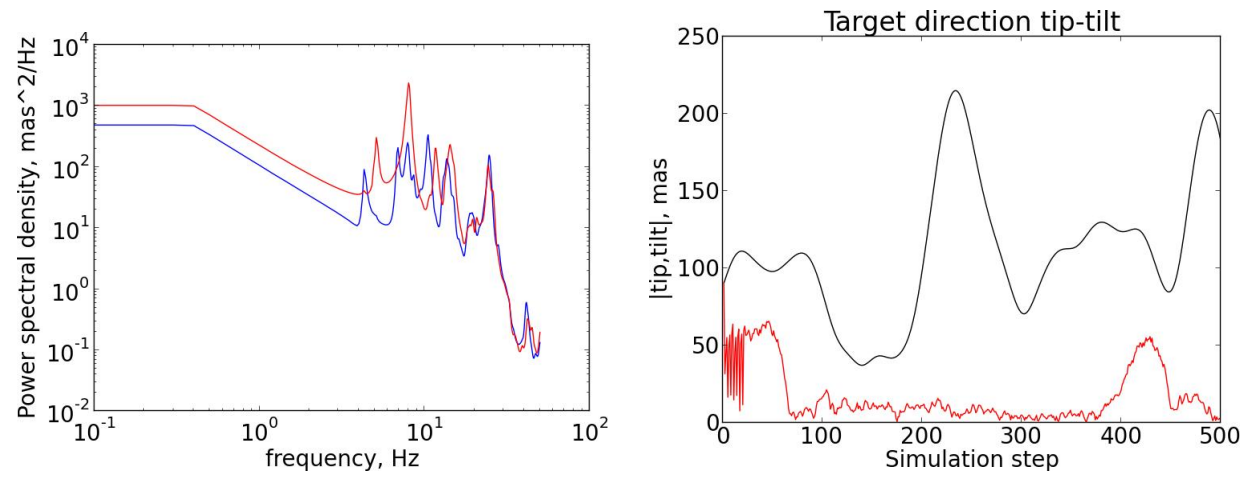

Figure 9. Left: Tip-tilt wind shake power spectral density modeled for GMT dome, xy-rms $=(57.2,38.9)$ mas, x-component is in blue, y-component is in red. Right: Evolution history of the random tip/tilt correction (wind shake + atmospheric tilt) in the tip-tilt sensor channel, integrator gain is 0.9 and sampling frequency is $1000 \mathrm{~Hz}$.

3. The focus control in the GMT LTAO system has three-fold purpose: 1) adjust to the LGS distance change as the telescope zenith angle changes from $0^{0}$ to $60^{\circ}, 2$ ) dynamically adjust the focus to changes in the LGS intensity distribution due to $\mathrm{Na}$ layer variability, 3) compensate the so called "LGS aberration" resulting from Shack-Hartmann WFS slope readout systematic error induced by LGS spot elongation because most of this error happens to be defocus (see Fig. 10).

4. The LGS stabilization channel (in fact 6 channels, one per each LGWFS) is needed to compensate the vibration- and turbulence-induced LGS beam wander to hold the star image within a small fraction of the sensor field of view. The feedback signal for this channel is the global tip/tilt extracted from each LGWFS, it is passed through a controller (integrator) to the tip/tilt DM installed into each LLT. Fig. 11 shows a time history of residual global tip/tilt on one of the LGWFSs obtained through an SL95 end-to-end simulation.

5. The pupil stabilization channel is used to reject motions of the exit pupil image on the LGWFS due to telescope structural perturbations. It can also provide the feedback signal for the pupil de-rotation. The principle of operation is based on balancing LGWFS peripheral subaperture (Fig. 12 a)) spot intensities 


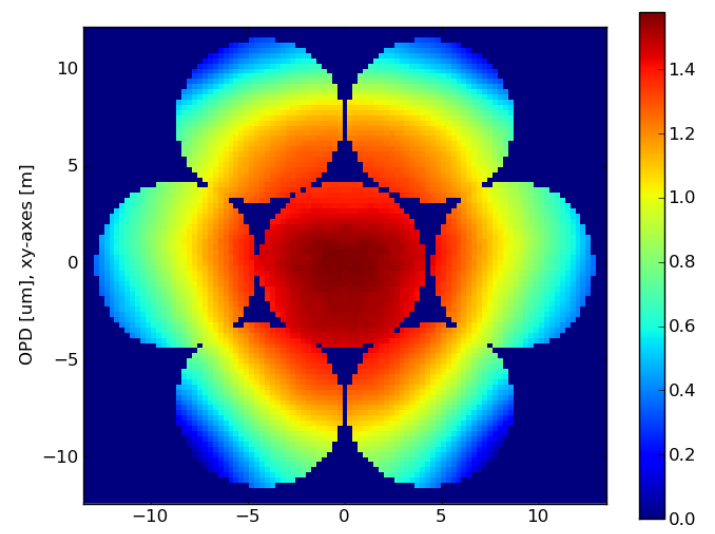

a)

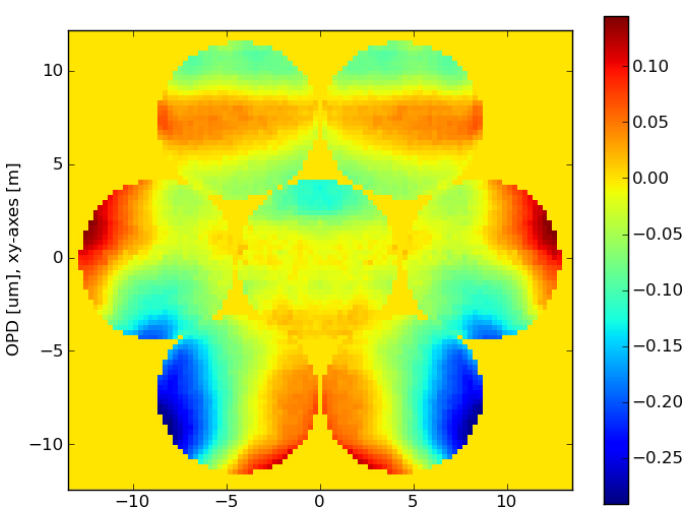

b)

Figure 10. LGS error illustration. a) Tomographic phase reconstruction from 6 LGWFSs referenced for point source but illuminated by extended LGS source (free propagation, no turbulence), RMS $=360 \mathrm{~nm}$. b) Same phase after focus removal, $\mathrm{RMS}=70 \mathrm{~nm}$.

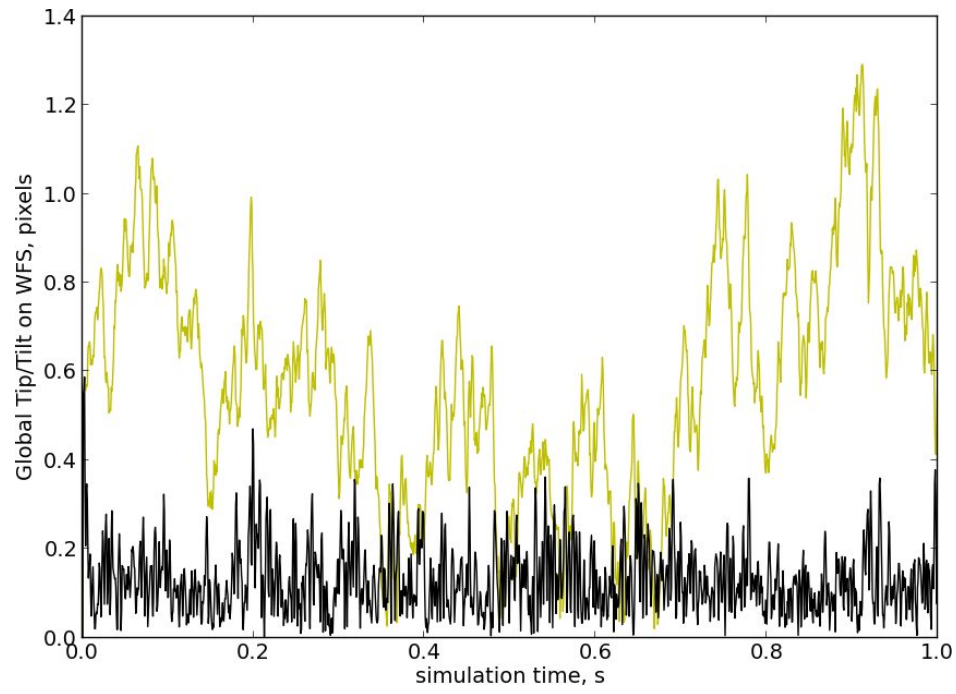

Figure 11. WFS global, open-loop tip/tilt time history without (yellow) and with (black) LLT beam stabilization at 1000 $\mathrm{Hz}$ sampling. The curves include beam up-link turbulence-induced wander, down-link turbulence contribution and also the sensor noise. 
that can be extracted from the sensor camera data flow. The intensity-invariant modification of the algorithm described in detail in Ref. 7 has small sensitivity to the sensor noise and scintillation as well as large dynamic range as illustrated on Fig. 12 b).
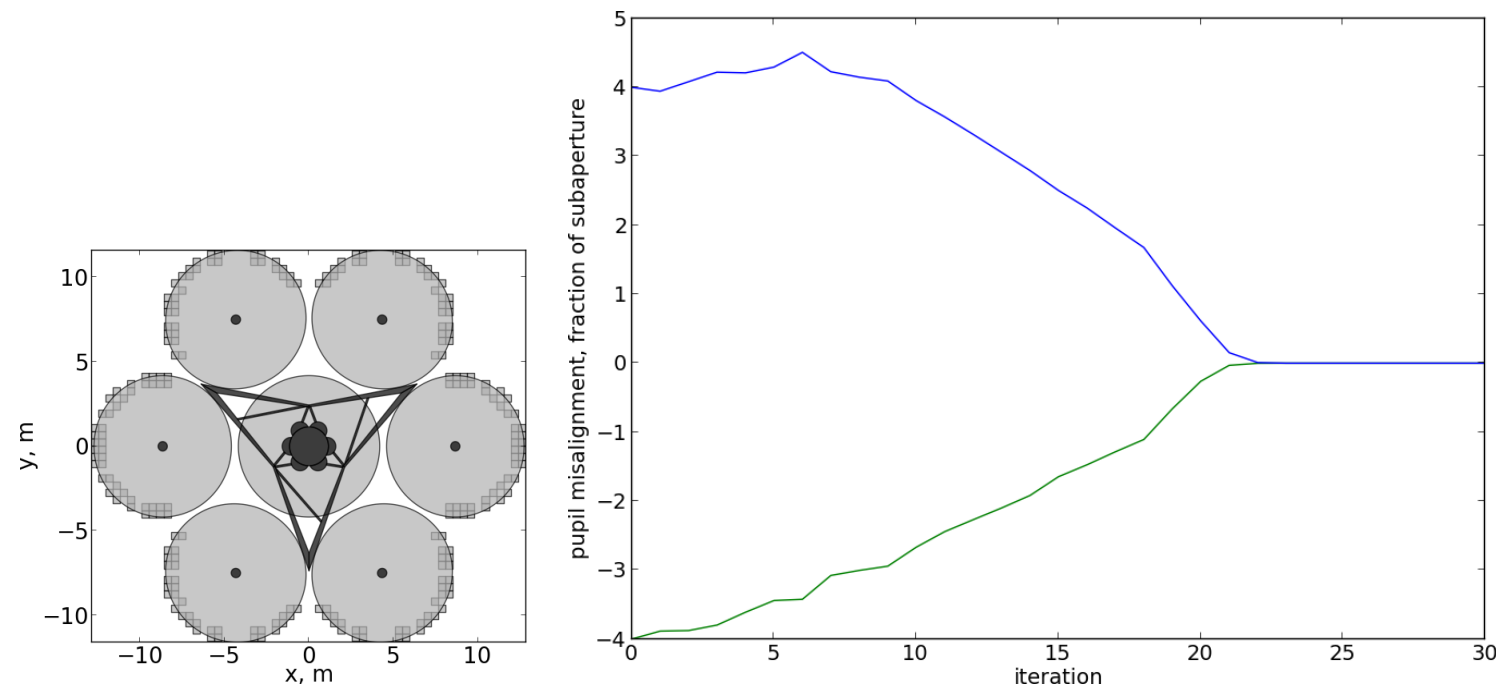

Figure 12. Left: peripheral subaperture pattern of the GMT LGWFS superimposed on the GMT segmented pupil. A total of 120 peripheral/reference subaperture pairs are chosen to provide signal to the pupil stabilization algorithm. Right: example of pupil position recovery from very large shift misalignment of 4 subaperture sizes in both $\mathrm{x}$ - and $\mathrm{y}$-directions. Green: x-misalignment. Blue: y-misalignment.

6. Truth sensor (TWFS) channel purpose is to measure and compensate the low order non-common path errors between the LGWFS and scientific instrument optical channels. A $16 \times 16$ truth sensor is planned to be installed in closed loop after ASM and OIDM, the reference source for it is the TT NGS albeit in the waveband different from the one used for the TTWFS. The non-common path aberration is reconstructed by a simple Least Squares single-conjugate reconstructor. Fig. 13 shows the TTWFS active subaperture map and the reconstructor errors due to turbulence residual after 10 second exposure.
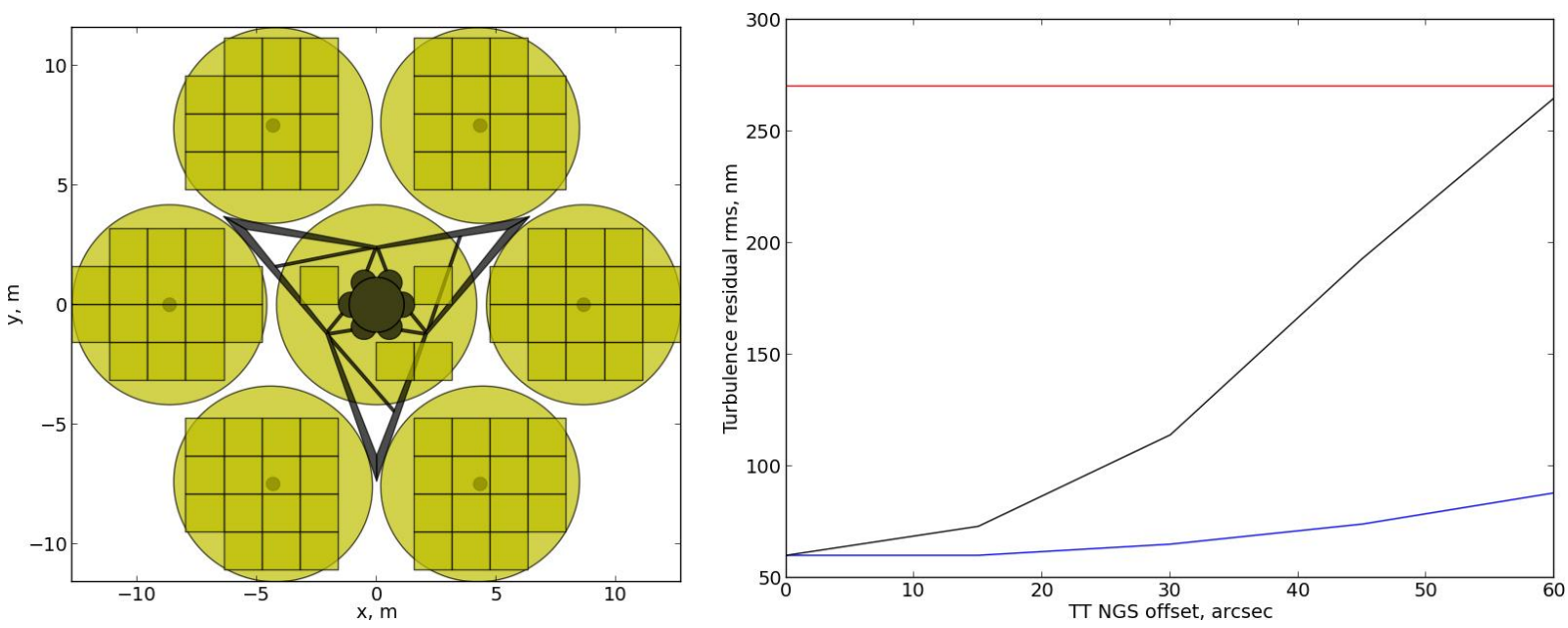

Figure 13. Left: Active subaperture map for the 16×16 GMT LTAO truth WFS. Right: Turbulence residual reconstruction error after 10 second exposure as a function of TT NGS offset. Blue: OIDM correction is on. Black: OIDM correction is off. Red: open-loop residual (no DM correction). 
7. Turbulence profile estimation channel uses the multiple sensor SLODAR technique. ${ }^{8}$ The main features of the SL95 SLODAR implementation are: theoretical models for atmospheric layer statistics, arbitrary layer altitude distribution, Toeplitz data compression, first or second readout differencing for tip/tilt and focus removal, ${ }^{9}$ arbitrary number of sensor inputs, positive-constrained Least-Squares solver. Fig. 14 demonstrates the result of the SLODAR turbulence profile estimation from the open-loop data taken from two LGWFS. The LTAO reconstructor based on this estimate gives performance indistinguishable from that of the reconstructor based on the known profile data.

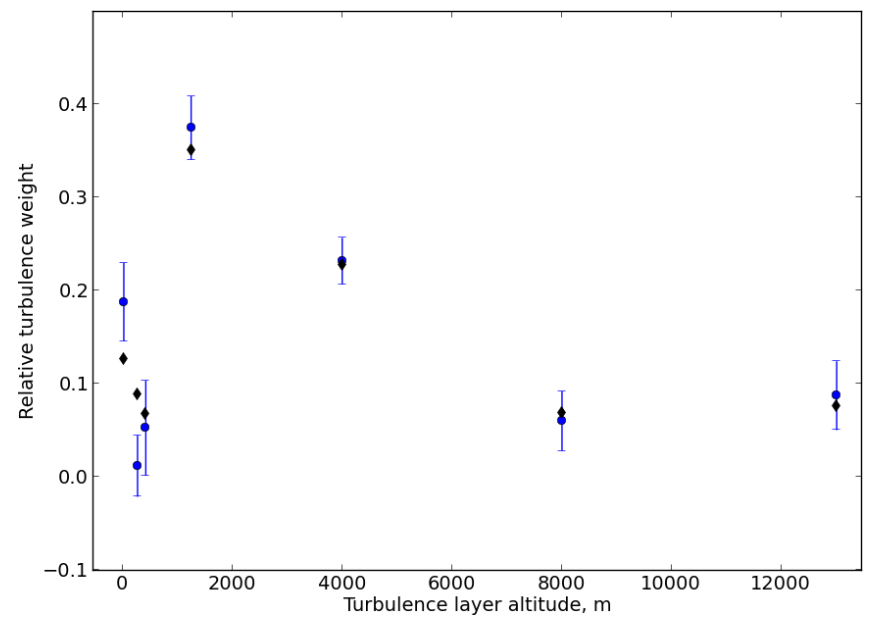

Figure 14. Estimation of atmosphere turbulence profile from LTAO SH WFSs readouts using the SLODAR technique. Open-loop operation, one pair of WFSs with 60" FoV offset, 10-second average with 25 ms intervals. Diamonds are the true turbulence weights of the standard GMT 7-layer turbulence profile. Dots and error bars are the SLODAR estimate with rms deviations obtained via Monte-Carlo simulation.

\section{SL95 STATUS AND FUTURE PLANS}

A list of the SL95 most important features, existing or planned, is presented in Table 1 to give a general idea of the feature set and its evolution in the future. The ultimate goal of the project is to create a simulation tool capable of integrated modeling of both active and adaptive systems that are responsible for complete wavefront control of extremely large telescopes and eventually provide the GMT project with a tool on a par with the ESO OCTOPUS package. ${ }^{11}$ 


\begin{tabular}{l|l}
\hline FEATURE & STATUS, comments \\
\hline System modeling and propagation & \\
Flat segmented optics, & ready \\
floating individual segment maps & \\
NGS and LGS models with photometry & ready \\
Geometrical propagation engine & ready \\
Wave propagation engine & under testing \\
LGS uplink propagation & ready \\
SH WFS model, & ready, \\
wave and geometrical internal & includes also \\
propagation, calibration & quad-cell TT-sensor \\
Pyramid WFS model, & planned \\
Atmospheric dispersion generator & planned \\
Fraticide generator & planned \\
Linear DM model, actuator interaction & ready \\
PSD- and Fractal-based & ready \\
turbulence generators & planned, based on Ref. 10 \\
3D optics ray tracing engine & planned \\
3D GMT optical model setup & planned \\
Real-time error modeling: & \\
camera rolling shutter, RTC jitter & ready \\
\hline Control & ready \\
SCAO Least Squares MVM controller \\
LTAO MV MVM controller & ready, published in Ref. 7 \\
Pupil stabilization controller & ready \\
SLODAR-based turbulence profiler & ready, leaky integrator \\
TT control channel & ready, leaky integrator \\
LGS stabilization channel & leaky integrator is inefficient, \\
Vibration control channel & adaptive LQG controller planned \\
& ready \\
Truth control channel & planned \\
Fast, non-MVM LTAO reconstructor & planned given the demand \\
MCAO, MOAO reconstructors & ready \\
\hline Simulation facilities & ready \\
Individual performance telemetry & ready, Matplotlib-based \\
for each system element & planned \\
Modular Monte-Carlo end-to-end & \\
simulation engine & \\
Data visualization system & \\
CPU-based parallelization & \\
\hline &
\end{tabular}

Table 1. SL95 feature matrix. 


\section{REFERENCES}

[1] Le Louarn, M., "Progress and prospects in AO simulation capabilities," Proc. SPIE 773618 (2010).

[2] Conan, R., Bennet, F., Bouchez, A., VanDam, M., Espeland, B., Gardhouse, R., d'Orgeville, C., Parcell, S., Piatrou, P., Price, I., Rigaut, F., Trancho, G., Uhlendorf, K., "The Giant Magellan Telescope Laser Tomography Adaptive Optics System," Proc. SPIE 8447-135, (2012).

[3] Johns, M., McCarthy, P. J., Raybould, K., Bouchez, A., Farahani, A., Filgueira, J. M., Jacoby, G., Shectman, S., Sheehan, M., "Giant Magellan Telescope: Overview," Proc. SPIE 8444, (2012).

[4] Decyk, V. K., Norton, C. D., Szymanski, B. K., "High performance object-oriented programming in Fortran 90," www.cs.rpi.edu/ szimansk/oof90.html.

[5] Pfrommer, T. and Hickson, P., "High-resolution mesospheric sodium observations for extremely large telescopes," Proc. SPIE 7736-773620 (2010).

[6] University of British Columbia, Large Zenith Telescope lidar data. http://142.103.239.59/lidar/archive.html

[7] Piatrou, P. and Chanan, G., "Shack-Hartmann mask/pupil registration algorithm for wavefront sensing in segmented mirror telescopes," Appl. Opt. 52, 7778-7784 (2013).

[8] Wang, L., Schöck, M., and Chanan, G., "Atmospheric turbulence profiling with SLODAR using multiple adaptive optics wavefront sensors," Appl. Opt., vol. 47 (2008).

[9] Gilles, L., Ellerbroek, B. L., "Real-time turbulence profiling with a pair of laser guide star Shack-Hartmann wavefront sensors for wide-field adaptive optics systems on large to extremely large telescopes," JOSA A, Vol. 27 (2010).

[10] Piatrou, P., "TMTracer: a modeling tool for the TMT alignment and phasing system," Proc. SPIE 8149, 814904 (2011).

[11] OCTOPUS: an end-to-end Adaptive Optics simulation tool of ESO. http://www.eso.org/sci/facilities/develop/ao/tecno.html 